\title{
Effects of damage-regulated autophagy regulator gene on the SGC7901 human gastric cancer cell line
}

\author{
BAO-SONG ZHU*, KUI ZHAO*, XIN JIA, YONG-YOU WU and CHUN-GEN XING \\ Department of General Surgery, The Second Affiliated Hospital, Soochow University, Suzhou, Jiangsu 215021, P.R. China
}

Received September 1, 2013; Accepted March 4, 2014

DOI: $10.3892 / \mathrm{ol} .2014 .2220$

\begin{abstract}
The aim of this study was to investigate the effects of the adenoviral-mediated autophagy gene, damage-regulated autophagy regulator(DRAM), on the proliferation andautophagy of SGC7901 human gastric cancer cells in vitro. The recombinant adenovirus, AdMax-pDC315-DRAM-EGFP, working as a virus vector of DRAM was constructed and infected into the SGC7901 human gastric cancer cell line. The MTT assay was used to determine the growth rate of the SGC7901 cells. Activation of autophagy was monitored with monodansylcadaverin(MDC) staining following AdMax-pDC315-DRAM-EGFP treatment. Immunofluorescent staining was used to examine the expression of microtubule-associated protein 1 light chain 3 (LC3), and western blotting was used to examine the expression of apoptosis- and autophagy-associated proteins, including Beclin1, p53, p21 and B-cell lymphoma 2 (Bcl-2), in the culture supernatant. The viability of the SGC7901 cells was activated by AdMax-pDC315-DRAM-EGFP treatment. The AdMax-pDC315-DRAM-EGFP-treated cells exhibited positive LC3 expression detected by immunoreactivity and MDC staining. Inductions in the expression of the apoptosis-related proteins, p53 and p21, and the autophagic protein, Beclin1, were revealed by western blot analysis. By contrast, downregulation of the apoptosis-related protein, Bcl-2, following AdMax-pDC315-DRAM-EGFP treatment was identified. In conclusion, the present study demonstrated that AdMax-pDC315-DRAM-EGFP treatment resulted in upregulation of the level of autophagy and induction of cell proliferation in the SGC7901 human gastric cancer cell line in vitro.
\end{abstract}

Correspondence to: Professor Chun-Gen Xing, Department of General Surgery, The Second Affiliated Hospital, Soochow University, 1055 Sanxiang Road, Suzhou, Jiangsu 215006, P.R. China E-mail: xingcg@126.com

*Contributed equally

Key words: damage-regulated autophagy regulator, p53, autophagy, cell proliferation

\section{Introduction}

Gastric cancer is the fourth most common type of cancer and the second leading cause of cancer-related mortality worldwide (1), with approximately one million new cases diagnosed each year. One of the major factors that controls tumor cell death is the tumor suppressor, p53 (2). The importance of cell death to tumor suppression is exemplified by p53 (3). In response to various forms of cellular stress, including DNA damage, hypoxia and oncogene activation, p53 levels are elevated (2). p53 has also been linked to another cell process that controls cell death known as autophagy $(4,5)$. Autophagy is a vesicular trafficking process that mediates the degradation of long-lived proteins and is the only pathway within the cell for the degradation of organelles (6). In tumor development, autophagy is considered to act in either an oncogenic or tumor suppressive capacity and p53 has been reported to be an inducer of autophagy $(4,5)$. Moreover, the discovery that damage-regulated autophagy regulator (DRAM), a p53 target gene which is required for p53-induced autophagy, is frequently downregulated in squamous cancers underscores the theory that autophagy is a component of tumor suppression downstream of p53 (5).

DRAM has been identified as an effector molecule that is critical for p53-mediated apoptosis, thus further supporting the tumor-suppressive role of autophagy $(5,7,8)$. The discovery of DRAM revealed a novel role for autophagy in p53-induced apoptotic cell death (5), and DRAM is considered to be a crucial modulator in apoptosis and autophagy. The present study aimed to investigate the effects of AdMax-pDC315-DRAM-EGFP on growth, apoptosis and autophagy of gastric cancer cells in vitro, and to compare the infection efficiency, biological and molecular mechanisms of AdMax-pDC315-DRAM-EGFP.

\section{Materials and methods}

Reagents. The SGC7901 gastric cancer cell line was purchased from the Shanghai Institute of Cell Biology, Chinese Academy of Sciences (Shanghai, China). The RPMI-1640 medium was purchased from Gibco-BRL (Rockville, MD, USA). Fetal bovine serum (FBS) was obtained from Hangzhou Sijiqing Biological Engineering Material Co., Ltd. (Hangzhou, China), and L-glutamine and MTT were provided by Sigma (St. Louis, MO, USA). Antibodies against p53 (1:500; Rabbit 
monoclonal anti-human), B cell lymphoma 2 (Bcl 2; 1:500; Rabbit monoclonal anti-human), Beclin1 (1:700; Rabbit monoclonal anti-human) and p21 (1;500; Rabbit monoclonal anti-human) were supplied by Cell Signaling Technology, Inc. (Beverly, MA, USA).

Adenoviral vectors and infections. The adenoviral vectors and NC-RNAi-GFP-AD were purchased from Shanghai Jikai Biological Technology Co., Ltd. (Shanghai, China). Stocks of replication-defective adenoviral vectors expressing green fluorescent protein (GFP) (AdMax-pDC315-DRAM-EGFP) were stored at $-80^{\circ} \mathrm{C}$. NC-RNAi-GFP-AD was used as a control which was also stored at $-80^{\circ} \mathrm{C}$. Infections were performed at $70-75 \%$ confluence in Dulbecco's modified Eagle's medium supplemented with $2 \%$ fetal calf serum (FCS). The cells were subsequently incubated at $37^{\circ} \mathrm{C}$ for at least $4 \mathrm{~h}$, followed by the addition of fresh medium. Cells were then subjected to functional analyses at fixed time points following infection as described for individual experimental conditions (9).

Determination of optimal multiplicity of infection (MOI). The SGC7901 cells $\left(1 \times 10^{4}\right.$ cells/well) were seeded in 96-well plates and reached $60-70 \%$ confluence. Different MOI $(\mathrm{MOI}=10,20$, 30,50 and 100) values of the NC-RNAi-GFP-AD 100- $\mu 1$ diluted infected cells were added to the plates and, after $8 \mathrm{~h}$, RPMI-1640 medium containing $10 \%$ FBS was added. After $48 \mathrm{~h}$ of culture, the cells were counted under a fluorescence microscope (Leica DMI4000B; Leica Microsystems Wetzlar GmbH, Wetzlar, Germany) to calculate the number of cells expressing GFP.

Cell culture and viability assay. The SGC7901 cells were maintained in RPMI-1640 medium containing 10\% heat-inactivated FBS and $0.03 \% \mathrm{~L}$-glutamine, and incubated in an atmosphere of $5 \% \mathrm{CO}_{2}$ at $37^{\circ} \mathrm{C}$. The cells in a mid-log phase were used in the experiments. Cell viability was assessed by the MTT assay. To determine the effects of AdMax-pDC315-DRAM-EGFP, the SGC7901 cells were plated into 96-well microplates (7x10 ${ }^{4}$ cells/well) and AdMax-pDC315-DRAM-EGFP was added to the culture medium. Cell viability was assessed by the MTT assay $24 \mathrm{~h}$ after AdMax-pDC315-DRAM-EGFP treatment. MTT (Sigma) solution was added to the culture medium (500 $\mu \mathrm{g} / \mathrm{ml}$ final concentration) for $4 \mathrm{~h}$ prior to the end of treatment and the reaction was inhibited by the addition of $10 \%$ acid sodium dodecyl sulfate (100 $\mu \mathrm{l}$; Beijing Biosea Biotechnology Co., Ltd., Beijing, China). The absorbance value (A) at $570 \mathrm{~nm}$ was measured using an automatic multi-well spectrophotometer (Bio-Rad, Richmond, CA, USA). The percentage of cell proliferation was calculated as follows: Cell proliferation $(\%)=(1-\mathrm{A}$ of experiment well/A of positive control well) x 100 .

Visualization of MDC-labeled vacuoles. Exponentially growing cells were plated on 24-chamber culture slides, cultured for $24 \mathrm{~h}$ and then incubated with the drug in 10\% FCS/RPMI-1640 medium for 12 and $24 \mathrm{~h}$. Autophagic vacuoles were labeled with MDC (Sigma) (10) by incubating cells with $0.001 \mathrm{mmol} / \mathrm{l} \mathrm{MDC}$ in RPMI- 1640 at $37^{\circ} \mathrm{C}$ for $10 \mathrm{~min}$. Following incubation, cells were washed three times with phosphate-buffered saline (PBS) and immediately analyzed with a fluorescence Nikon Eclipse TE300 microscope (Nikon, Tokyo, Japan) equipped with a filter system (V-2A excitation filter, 380-420 nm; barrier filter,

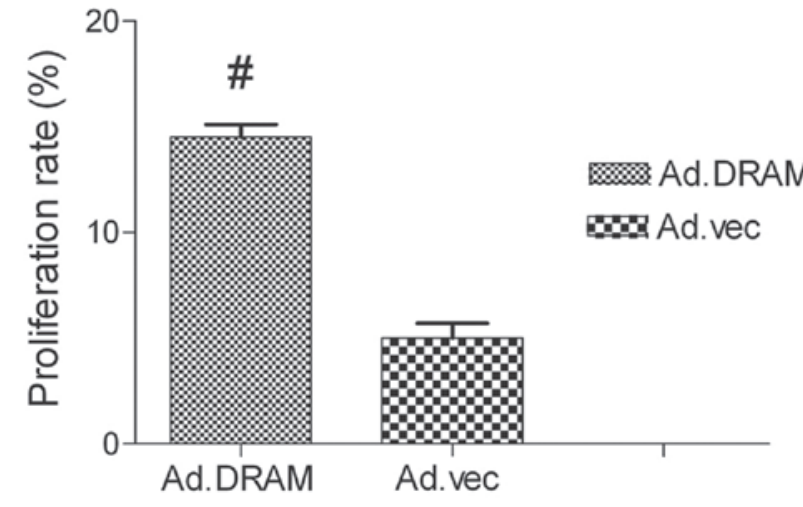

Figure 1. Reduced cell viability following AdMax-pDC315-DRAM-EGFP treatment. SGC7901 cells $\left(7 \times 10^{4}\right.$ cells $\left./ \mathrm{ml}\right)$ were cultured with AdMax-pDC315-DRAM-EGFP (MOI, 60) for $24 \mathrm{~h}$ and cell viability was analyzed by the MTT assay. Values are expressed as the mean \pm standard deviation of three independent experiments. ${ }^{\#} \mathrm{P}<0.05$, compared with the control group. DRAM, damage-regulated autophagy regulator; MOI, multiplicity of infection; vec, vector.

$450 \mathrm{~nm}$ ). Images were captured with a charged couple device camera (CoolSNAP ES, Roper Scientific; Trenton, NJ, USA) and imported into Photoshop.

Immunofluorescent staining. The SGC7901 cells were seeded onto 24-chamber culture slides and treated with AdMax-pDC315-DRAM-EGFP. Following fixation in methanol for 10 min, cells were blocked with a buffer containing $1 \%$ bovine serum albumin (BSA; Hangzhou Sijiqing Biological Engineering Material Co., Ltd.) and 0.1\% Triton X-100 (Nanjing KeyGen Biotech., Co., Ltd., Nanjing, China) for $1 \mathrm{~h}$. The cells were then incubated with the primary antibody against LC3 (diluted 1:200; Santa Cruz Biotechnology, Inc., Santa Cruz, CA, USA) and PBS containing $1 \% \mathrm{BSA}$ at $4^{\circ} \mathrm{C}$ overnight, and then incubated for $1 \mathrm{~h}$ with secondary ghost against rabbit cy3 fluorescence conjugated antibodies (1:500; Sigma) to visualize the binding sites of the primary antibody with laser confocal microscopy (Leica Microsystems Wetzlar GmbH).

Total cell protein extraction and western blot analysis. For extraction of total cell proteins, cells were washed with pre-cooled PBS and subsequently lysed in pre-cooled radioimmunoprecipitation assay lysis buffer $[50 \mathrm{mM}$ Tris- $\mathrm{HCl}(\mathrm{pH} 7.4)$, $150 \mathrm{mM} \mathrm{NaCl}, 1 \mathrm{mM}$ dithiothreitol, $0.25 \%$ sodium deoxycholate and $0.1 \% \mathrm{NP}-40$ ] containing $1 \mathrm{mM}$ phenylmethysulfonyl fluoride, $50 \mathrm{mM}$ sodium pyrophosphate, $1 \mathrm{mM} \mathrm{Na}_{3} \mathrm{VO}_{4}, 1 \mathrm{mM}$ $\mathrm{NaF}, 5 \mathrm{mM}$ EDTA, $5 \mathrm{mM}$ EGTA and protease inhibitors cocktail (Nantong Biyuntian Biological Technology Co., Ltd., Nantong,China). Cell lysis was performed on ice for $30 \mathrm{~min}$. Clear protein extracts were obtained by centrifugation $12,000 \mathrm{xg}$ for $30 \mathrm{~min}$ at $4^{\circ} \mathrm{C}$. Protein extraction from the SGC7901 gastric cancer cells was performed as previously described (11). Protein concentration was determined with a Bradford protein assay kit (Nanjing KeyGen Biotech., Co., Ltd.). Proteins were resolved on $8.5 \%$ polyacrylamide gels (Nantong Biyuntian Biological Technology Co., Ltd.) and subsequently transferred onto nitrocellulose membranes (Nanjing KeyGen Biotech., Co., Ltd.). For immunoblotting, nitrocellulose membranes were incubated with specific antibodies recognizing target proteins overnight 

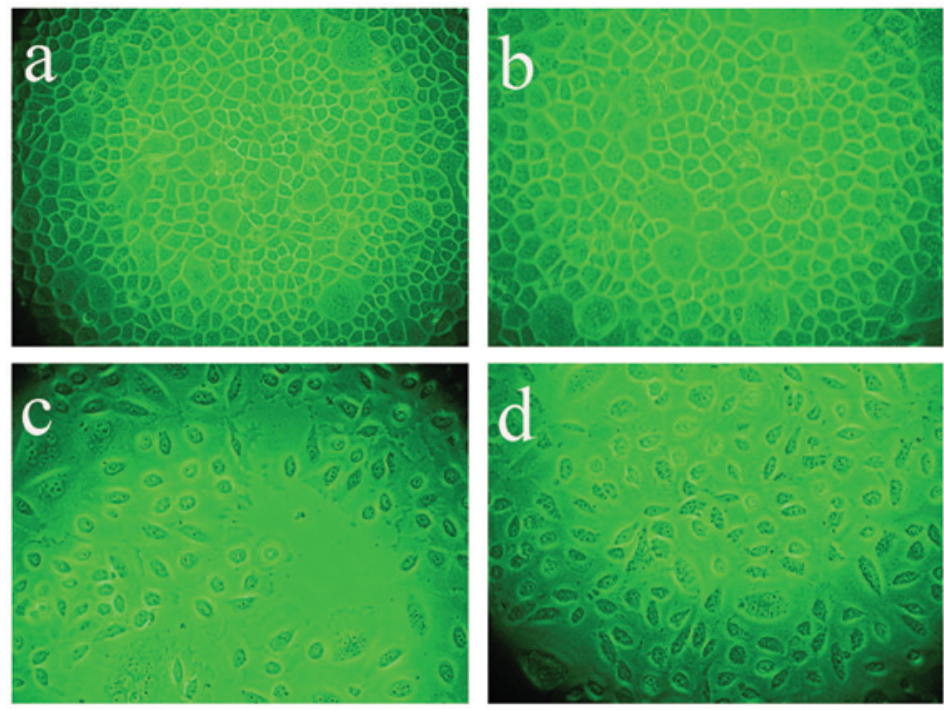

Figure 2. Infection efficiency and cell morphology were analyzed under a fluorescence microscope following AdMax-pDC315-DRAM-EGFP (MOI, 60) and AdMax-pDC315-EGFP (MOI, 60) treatment. The SGC7901 cells were incubated with AdMax-pDC315-DRAM-EGFP (MOI, 60) for the indicated time. (A) Control, (B) AdMax-pDC315-EGFP, (C) $12 \mathrm{~h}$ after AdMax-pDC315-DRAM-EGFP (MOI, 60) treatment and (D) $24 \mathrm{~h}$ after AdMax-pDC315-DRAM-EGFP (MOI, 60) treatment. Magnification, x200. DRAM, damage-regulated autophagy regulator; MOI, multiplicity of infection.
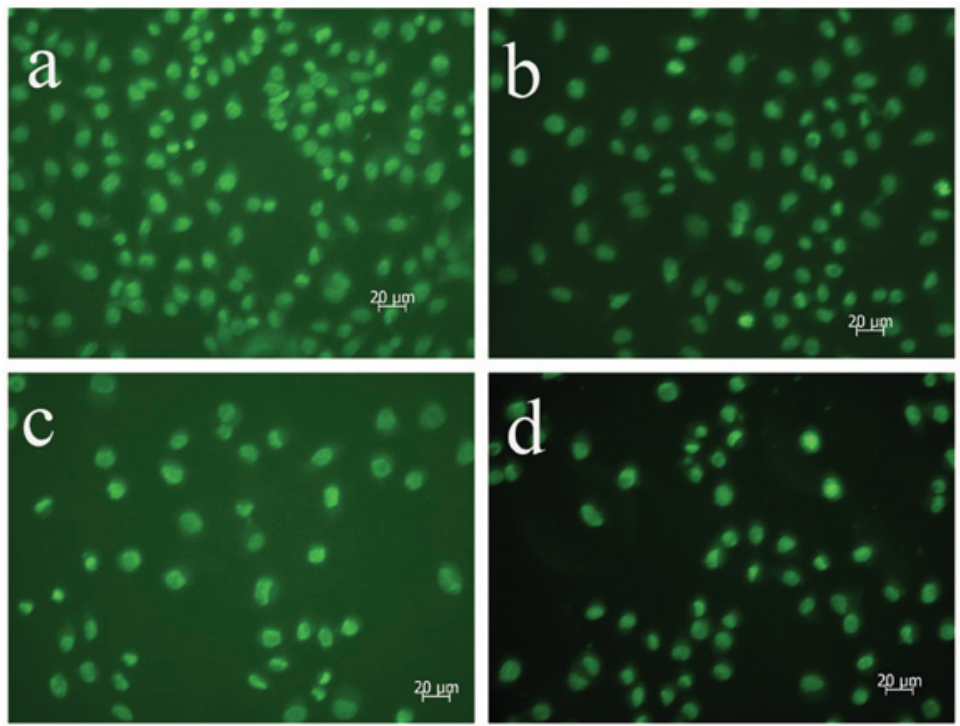

Figure 3. MDC staining revealed autophagy was activated following AdMax-pDC315-DRAM-EGFP (MOI, 60) and AdMax-pDC315-EGFP (MOI, 60) treatment. The SGC7901 cells were incubated with AdMax-pDC315-DRAM-EGFP (MOI, 60) for an indicated time and stained with MDC (100 $\mu$ mol/1). Fluorescent particles revealed late autophagic vacuoles. (A) Control, (B) AdMax-pDC315-EGFP, (C) $12 \mathrm{~h}$ after AdMax-pDC315-DRAM-EGFP (MOI, 60) treatment and (D) $24 \mathrm{~h}$ after AdMax-pDC315-DRAM-EGFP (MOI, 60) treatment. Magnification, x1,000. MDC, monodansylcadaverin; MOI, multiplicity of infection; DRAM, damage-regulated autophagy regulator.

at $4^{\circ} \mathrm{C}$. The membranes were washed as previously described and then incubated with horseradish peroxidase-conjugated goat anti-rabbit IgG monoclonal secondary antibody (1:20,000; Amersham Pharmacia Biotech, Arlington Heights, IL, USA) for $1 \mathrm{~h}$ at room temperature and and visualized by autoradiograpy. $\beta$-actin protein (1:5,000; Sigma) was used as the loading control. The membrane was washed three times with Tris-buffered saline and Tween 20 [10 mM Tris- $\mathrm{HCl}$ (pH 8.0), $150 \mathrm{mM} \mathrm{NaCl}$ and $0.5 \%$ Tween-20] and developed using the enhanced chemiluminescence detection system (Amersham Pharmacia Biotech). The intensity of the immunoreactive bands was quantified using a densitometer (SI, Molecular Dynamics, Sunnyvale, CA, USA).
Statistical analysis. All data are presented as the mean \pm standard deviation. Statistical analysis was performed by analysis of variance followed by Dunnett's test. $\mathrm{P}<0.05$ was considered to indicate a statistically significant difference.

\section{Results}

AdMax-pDC315-DRAM-EGFP treatment increases cell viability. The MTT assay showed that the proliferation capacity of gastric cancer cells infected with AdMax-pDC315-DRAM-EGFP was significantly higher than AdMax-pDC315-EGFP (MOI, 60) $(\mathrm{P}<0.05)$. The DRAM gene 

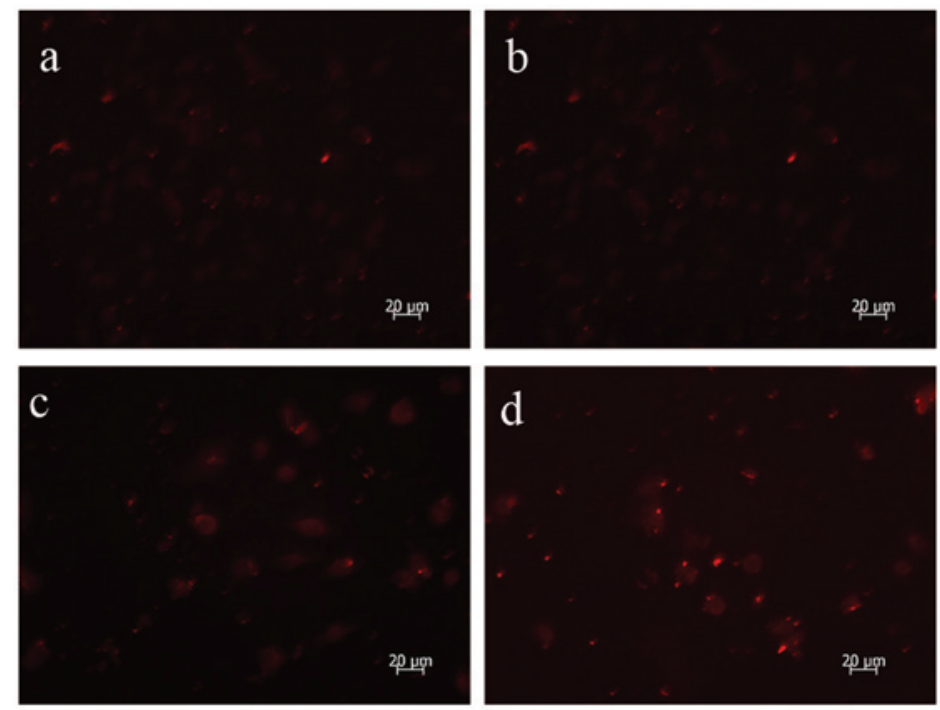

Figure 4. Microtubule-associated protein 1 light chain 3 expression and location in SGC7901 cells following treatment with AdMax-pDC315-DRAM-EGFP. (A) Control and (B) AdMax-pDC315-EGFP control (magnification, $x 1,000)(n=3)$. Cells were treated with AdMax-pDC315-DRAM-EGFP (multiplicity of infection, 60) for (C) 12 and (D) $24 \mathrm{~h}$, and observed by immunofluorescence microscopy. AdMax-pDC315-DRAM-EGFP increased the punctuate distribution of LC3 from 12 to $24 \mathrm{~h}$. DRAM, damage-regulated autophagy regulator.
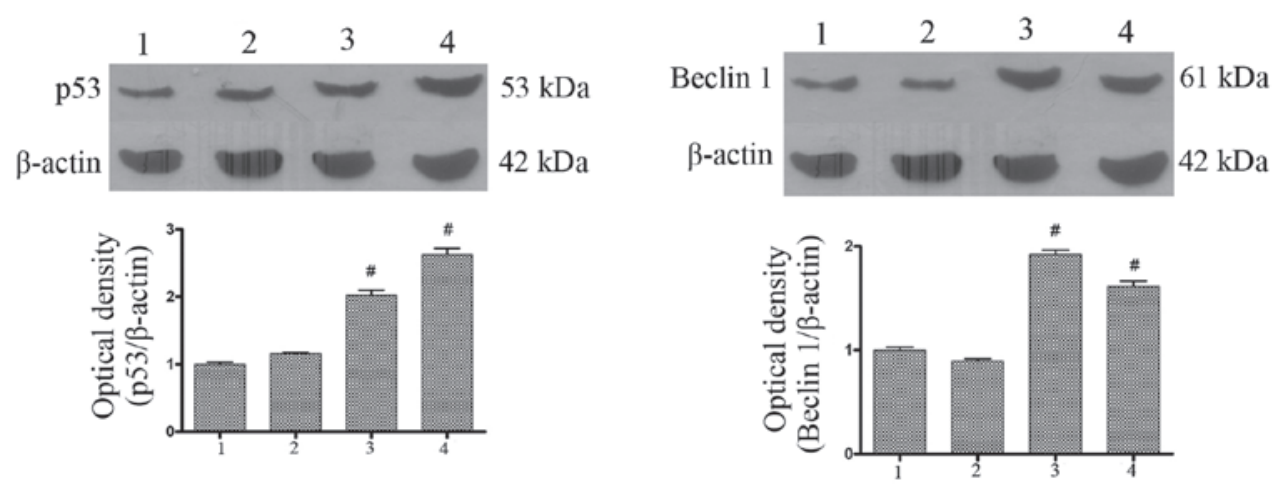

Figure 5. Effect of AdMax-pDC315-DRAM-EGFP (MOI, 60) infection on p53 and Beclin1 protein expression. The SGC7901 cells were treated with AdMax-pDC315-DRAM-EGFP (MOI, 60) and AdMax-pDC315-EGFP (MOI, 60) for 12 and $24 \mathrm{~h}$, then harvested for extraction of total proteins. AdMax-pDC315-DRAM-EGFP upregulated the expression of p53 and Beclin1 protein. 1, normal group; 2, AdMax-pDC315-EGFP group; 3 , AdMax-pDC315-DRAM-EGFP treatment for $12 \mathrm{~h}$; 4, AdMax-pDC315-DRAM-EGFP treatment for $24 \mathrm{~h}$. Statistical comparisons were performed using Dunnett's test $(\mathrm{n}=3)$. Values are expressed as the mean \pm standard deviation. ${ }^{~} \mathrm{P}<0.01$, compared with the control group. Cont, control; DRAM, damage-regulated autophagy regulator; MOI, multiplicity of infection.
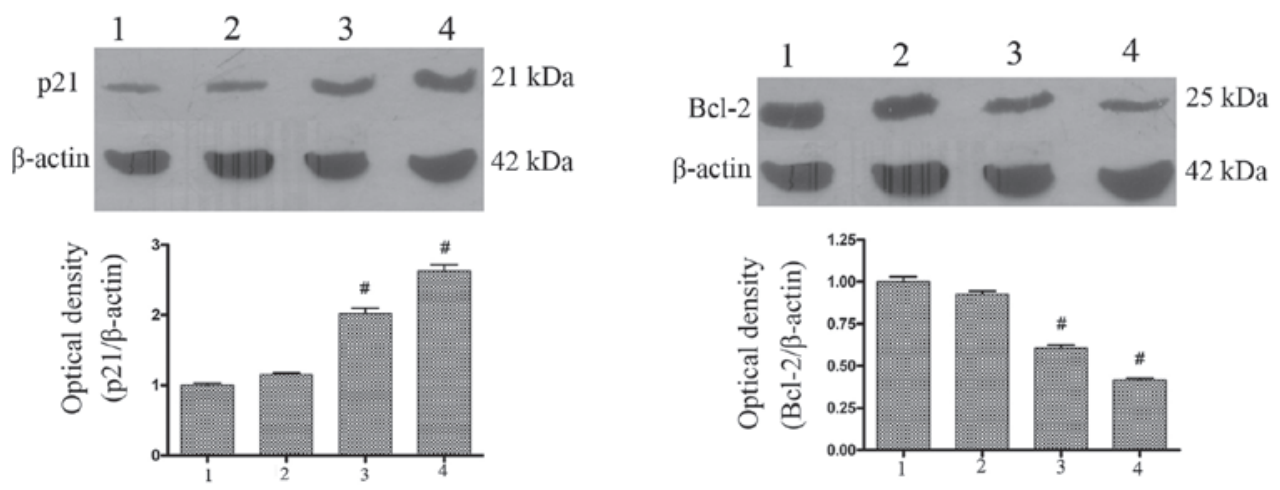

Figure 6.Effects of AdMax-pDC315-DRAM-EGFP infection on Bcl-2 and 21 protein expression in SGC7901 cells.(A) Effects of AdMax-pDC315-DRAMEGFP (MOI, 60) on Bcl-2 and p21 protein expression. The SGC7901 cells were treated with AdMax-pDC315-DRAM-EGFP (MOI, 60) and AdMax-pDC315-EGFP (MOI, 60) for 12 or $24 \mathrm{~h}$ then harvested for extraction of total proteins. AdMax-pDC315-DRAM-EGFP upregulated the expression of p21 and downregulated the expression of Bcl-2 protein. 1, normal group; 2, AdMax-pDC315-EGFP group; 3, AdMax-pDC315-DRAM-EGFP treatment for 12 h; 4 , AdMax-pDC315-DRAM-EGFP treatment for $24 \mathrm{~h}$. Statistical comparisons were performed using Dunnett's test ( $\mathrm{n}=3$ ). Values are expressed as the mean \pm standard deviation. "P<0.01, compared with the control group. Cont, control; DRAM, damage-regulated autophagy regulator; Bcl-2, B-cell lymphoma 2; MOI, multiplicity of infection. 
promoted the proliferation of cell viability. After $24 \mathrm{~h}$ of treatment, the rate of proliferation had reached $14.71 \pm 4.13 \%$ (Fig. 1).

Infection efficiency and cell morphology. Following AdMax-pDC315-DRAM-EGFP (MOI, 60) infection (12 h) of SGC7901 cells, the cell body appeared swollen, rounded and the cells revealed deformation, with the cells showing further deformation after $24 \mathrm{~h}$. After infection for $24 \mathrm{~h}$, the SGC7901 cells were counted under a fluorescence microscope to determine the percentage of the of infected cells (Fig. 2). Infection efficiency did not increase with increasing MOI and the time of infection. The infection efficiency and cell viability are dependent on the correct MOI (60) and the time of infection (24 h). It was determined that at an MOI of 60 , the infection efficiency was $93 \pm 5.4 \%$.

AdMax-pDC315-DRAM-EGFP infection increases autophagic vacuoles. The autofluorescent substance, MDC, is a marker for late autophagic vacuoles (L-AVs), but not endosomes (12). The dye is trapped in acidic, membrane-rich organelles and exhibits an increased fluorescence quantum yield in response to the compacted lipid bilayers present in L-AVS (10). When cells are analyzed under a fluorescent microscope, AVs stained by MDC appear as distinct dot-like structures distributed within the cytoplasm or localizing in the perinuclear regions. In this study, an increase in the number of MDC-labeled vesicles following infection with AdMax-pDC315-DRAM-EGFP (MOI, 60) from 12 to 24 h was observed (Fig. 3).

AdMax-pDC315-DRAM-EGFP infection upregulates the expression of LC3. Microtubule-associated protein $1 \mathrm{LD} 3$, the mammalian ontology of Atg8, targets to the autophagosomal membranes in an Atg5-dependent manner and remains there even after Atg12-Atg5 dissociates. LC3 is considered to be the only credible marker of the autophagosome in mammalian cells (13). The present study used immunofluorescence to analyze the expression and location of LC3 and identified an increased formation of autophagosomes following AdMax-pDC315-DRAM-EGFP (MOI, 60) infection (Fig. 4).

AdMax-pDC315-DRAM-EGFP infection upregulates the expression of Beclinl and p53. To investigate the effects of AdMax-pDC315-DRAM-EGFP (MOI, 60) infection on the expression of autophagic-related proteins, western blot analysis was used to detect the expression of p53 and Beclin1. The findings revealed that the basal level of Beclin1 and p53 in the SGC7901 cells was low. Following incubation with AdMax-pDC315-DRAM-EGFP (MOI, 60), the Beclin1 and p53 protein expression levels significantly increased from 12 to $24 \mathrm{~h}$ (Fig. 5).

AdMax-pDC315-DRAM-EGFP infection increases the expression of $p 21$ and decreases the expression of $\mathrm{Bcl}-2$. To determine whether AdMax-pDC315-DRAM-EGFP (MOI, 60) infection affects the expression of apoptotic-related proteins, western blot analysis was used to detect the expression of Bcl-2 and p21 (Fig. 6). The findings revealed that the basal level of p21 protein in SGC7901 cells was low; however, following incubation with DRAM, the p21 protein expression levels were significantly increased from 12 to $24 \mathrm{~h}$. By contrast, the $\mathrm{Bcl}-2$ protein expression levels were downregulated with the addition of AdMax-pDC315-DRAM-EGFP (MOI, 60) (Fig. 6).

\section{Discussion}

A number of studies have found that the baseline levels of autophagy act as a tumor suppressor mechanism. Nevertheless, stress-induced autophagy constitutes a major pro-survival mechanism for tumors exposed to a hypoxic microenvironment or to chemotherapeutic agents. Thus, autophagy mediates either antitumor or pro-tumor functions $(14,15)$, and hence, is considered as a 'double-edged sword' in oncogenesis and tumor progression (16).

The genetic inactivation of $\mathrm{p} 53$, the best-known human oncosuppressor protein, has been observed in $>50 \%$ of all types of human cancer and mostly mediates tumor suppression, not only by transactivating pro-apoptotic and cell cycle arresting genes, but also by regulating autophagy. p53 mutations that simultaneously abolish its pro-apoptotic and autophagy-inhibitory functions behave as 'multi-hit' events, as opposed to 'single-hit' mutations that only affect the classical (pro-apoptotic and/or cell cycle-arresting) functions of the p53 system $(17,18)$.

Under genotoxic stress, p53 has been shown to upregulate the transcription of DRAM. DRAM, a 238-amino acid protein, which is highly conserved in higher eukaryotes, is localized to the lysosomal membrane. Knockdown of DRAM expression promoted survival following exposure to DNA-damage, and DRAM is also required for p53-induced autophagy and cell death (5).

In the present study, the autophagic level is low in the SGC7901 gastric cancer cell line; however, with the addition of DRAM adenovirus the autophagy-specific marker, LC3, was upregulated indicating an increased formation of autophagosomes induced by DRAM infection. Beclin1, the mammalian ortholog of the yeast apg6/vps30 gene, plays a role in two fundamentally important cell biological pathways, autophagy and apoptosis. Beclin1 is a major determinant in the initiation of autophagy (18-21).

Beclin1 is monoallelically deleted in human breast and ovarian cancers and is expressed at reduced levels in those tumors $(22,23)$. The findings of the present study suggest that autophagy is induced by DRAM and its activation may not contribute to the antitumor effects of DRAM. Moreover, DRAM increased the expression of Beclin1, particularly the production of $\mathrm{p} 53 . \mathrm{Bcl}-2$ and $\mathrm{Bcl}-\mathrm{xL}$ are associated with the evolutionarily conserved autophagy inducer, Beclin1, a haplo-insufficient tumor suppressor (24), and inhibit autophagy (25). The inhibition may require Bcl-2 to localize on the endoplasmic reticulum $(25,26)$ and, notably, the $\mathrm{BH} 3$ domain of Beclin1 mediates their association (27). In the present study, the expression of Beclin1 was upregulated with the treatment of DRAM and the expression of Bcl-2 was decreased, indicating that DRAM may have decreased the expression of Bcl-2. The cell-cycle-regulating protein, $\mathrm{p} 21$, is a cyclin-dependent kinase inhibitor coupled to a wide variety of cell functions, including p53-dependent growth suppression, cell cycle arrest following DNA damage, and the inhibition and induction of apoptosis (28). In our study, following DRAM 
infection, the expression of p53 was upregulated simultaneously to the increase of $\mathrm{p} 21$.

In conclusion, the level of autophagy increased with the addition of DRAM, which also induced proliferation of the SGC7901 cells. The integrative effect of autophagy induced by DRAM may have activated the proliferation of the SGC7901 cells. These findings suggest that autophagy induces the survival of the SGC7901 tumor cell line. Further study should investigate the effects of DRAM on primary culture gastric cancer cells collected from patients with gastric cancer. This may provide novel treatment strategies for patients with gastric cancer.

\section{Acknowledgements}

This study was supported by the Natural Science Foundation of China (grant no. 81172348) and the Suzhou Science and Technology Development Foundation (grant nos. 2010SYS201031 and 2011SYSD2011092).

\section{References}

1. Crew KD and Neugut AI: Epidemiology of gastric cancer. World J Gastroenterol 12: 354-362, 2006.

2. Crighton D and Ryan KM: Splicing DNA-damage responses to tumor cell death. Biochim Biophys Acta 1705: 3-15, 2004.

3. Ryan KM, Phillips AC and Vousden KH: Regulation and function of the p53 tumor suppressor protein. Curr Opin Cell Biol 13: 332-337, 2001.

4. Feng Z, Zhang H, Levine AJ and Jin S: The coordinate regulation of the p53 and mTOR pathways in cells. Proc Natl Acad Sci USA 102: 8204-8209, 2005.

5. Crighton D, Wilkinson S, O'Prey J, Syed N, Smith P, Harrison PR, Gasco M, Garrone O, Crook T and Ryan KM: DRAM, a p53-induced modulator of autophagy, is critical for apoptosis. Cell 126: 121-134, 2006.

6. Klionsky DJ and Emr SD: Autophagy as a regulated pathway of cellular degradation. Science 290: 1717-1721, 2000.

7. Kerley-Hamilton JS, Pike AM, Hutchinson JA, Freemantle SJ and Spinella MJ: The direct p53 target gene, FLJ11259/DRAM, is a member of a novel family of transmembrane proteins. Biochim Biophys Acta 1769: 209-219, 2007.

8. Crighton D, O'Prey J, Bell HS and Ryan KM: p73 regulates DRAM-independent autophagy that does not contribute to programmed cell death. Cell Death Differ 14: 1071-1079, 2007.

9. Alesci S, Ramsey WJ, Bornstein SR, Chrousos GP, Hornsby PJ, Benvenga S, Trimarchi F and Ehrhart-Bornstein M: Adenoviral vectors can impair adrenocortical steroidogenesis: clinical implications for natural infections and gene therapy. Proc Natl Acad Sci USA 99: 7484-7489, 2002.

10. Niemann A, Takatsuki A and Elsässer HP: The lysosomotropic agent monodansylcadaverine also acts as a solvent polarity probe. J Histochem Cytochem 48: 251-258, 2000.

11. Ikeda K, Monden T, Kanoh T, Tsujie M, Izawa H, Haba A, Ohnishi T, Sekimoto M, Tomita N, Shiozaki H and Monden M: Extraction and analysis of diagnostically useful proteins from formalin-fixed, paraffin-embedded tissue sections. J Histochem Cytochem 46: 397-403.
12. Biederbick A, Kern HF and Elsässer HP: Monodansylcadaverine (MDC) is a specific in vivo marker for autophagic vacuoles. Eur J Cell Biol 66: 3-14, 1995.

13. Yoshimori T: Autophagy: a regulated bulk degradation process inside cells. Biochem Biophys Res Commun 313: 453-458, 2004.

14. Maiuri MC, Tasdemir E, Criollo A, Morselli E, Vicencio JM, Carnuccio R and Kroemer G. Control of autophagy by oncogenes and tumor suppressor genes. Cell Death Differ 16: 87-93, 2009.

15. Morselli E, Galluzzi L, Kepp O, Vicencio JM, Criollo A, Maiuri MC and Kroemer G: Anti- and pro-tumor functions of autophagy. Biochim Biophys Acta 1793: 1524-1532, 2009.

16. White E and DiPaola RS: The double-edged sword of autophagy modulation in cancer. Clin Cancer Res 15: 5308-5316, 2009.

17. Soussi T: p53 alterations in human cancer: more questions than answers. Oncogene 26: 2145-2156, 2007.

18. Soussi T and Lozano G: p53 mutation heterogeneity in cancer. Biochem Biophys Res Commun 331: 834-842, 2005.

19. Liang XH, Jackson S, Seaman M, Brown K, Kempkes B, Hibshoosh $\mathrm{H}$ and Levine B: Induction of autophagy and inhibition of tumorigenesis by beclin 1. Nature 402: 672-676, 1999.

20. Yue Z, Jin S, Yang C, Levine AJ and Heintz N: Beclin 1, an autophagy gene essential for early embryonic development, is a haploinsufficient tumor suppressor. Proc Natl Acad Sci USA 100: $15077-15082,2003$

21. Zeng X, Overmeyer JH and Maltese WA: Functional specificity of the mammalian Beclin-Vps34 PI 3-kinase complex in macroautophagy versus endocytosis and lysosomal enzyme trafficking. J Cell Sci 119: 259-270, 2006.

22. Lum JJ, DeBerardinis RJ and Thompson CB: Autophagy in metazoans: cell survival in the land of plenty (review). Nat Rev Mol Cell Biol 6: 439-448, 2005.

23. Aita VM, Liang XH, Murty VV, Pincus DL, Yu W, Cayanis E, Kalachikov S, Gilliam TC and Levine B: Cloning and genomic organization of Beclin 1, a candidate tumor suppressor gene on chromosome 17q21. Genomics 59: 59-65, 1999.

24. Qu X, Yu J, Bhagat G, Furuya N, Hibshoosh H, Troxel A, Rosen J, Eskelinen EL, Mizushima N, Ohsumi Y, Cattoretti G and Levine B: Promotion of tumorigenesis by heterozygous disruption of the beclin 1 autophagy gene. J Clin Invest 112: 1809-1820, 2003.

25. Pattingre S, Tassa A, Qu X, Garuti R, Liang XH, Mizushima N, Packer M, Schneider MD and Levine B: Bcl-2 antiapoptotic proteins inhibit Beclin 1-dependent autophagy. Cell 122: 927-939, 2005.

26. Hoyer-Hansen M, Bastholm L, Szyniarowski P, Campanella M, Szabadkai G, Farkas T, Bianchi K, Fehrenbacher N, Elling F, Rizzuto R, Mathiasen IS and Jäättelä M: Control of macroautophagy by calcium, calmodulindependent kinase kinase-beta, and Bcl-2. Mol Cell 25:193-205, 2007.

27. Oberstein A, Jeffrey PD and Shi Y: Crystal structure of the Bcl-XL-Beclin 1 peptide complex: Beclin 1 is a novel BH3-only protein. J Biol Chem 282: 13123-13132, 2007.

28. Le NT and Richardson DR: Potent iron chelators increase the mRNA levels of the universal cyclin-dependent kinase inhibitor p21(CIP1/WAF1), but paradoxically inhibit its translation: a potential mechanism of cell cycle dysregulation. Carcinogenesis 24 : 1045-1058, 2003. 